\section{Estrategias del aprendizaje inicial de la clínica: más allá de los hábitos de estudio. Percepciones de estudiantes y docentes clínicos}

\author{
DENISSE ZÚÑIGA ${ }^{1, \mathrm{a}}$, ISABEL LEIVA², MARIBEL CALDERÓN,c, \\ ALEMKA TOMICIC ${ }^{4, \mathrm{~d}}$, OSLANDO PADILLA ${ }^{5, \mathrm{e}}$, \\ ARNOLDO RIQUELME ${ }^{6}$, MARCELA BITRAN ${ }^{1, b}$
}

\section{Learning during the early clinical years takes more than good study habits. Perceptions of students and teachers}

Background: Teaching methods of the undergraduate medical curriculum change considerably from the first years to clinical training. Clinical learning occurs in complex and varied scenarios while caring for patients. Students have to adapt their learning approaches and strategies to be able to integrate theory and clinical practice and become experiential learners. Aim: To identify the strategies used by medical students to learn during the initial clinical years, as reported by students themselves and by their clinical tutors. Material and Methods: We performed eight focus group discussions with 54 students enrolled in years three to six and we interviewed eight clinical tutors. Both focus group discussions and interviews were audio recorded, transcribed and analyzed according to Grounded Theory. Results: Four main themes were identified in the discourse of both students and tutors: Strategies oriented to theoretical learning, strategies oriented to experiential learning, strategies for integrating theory and practice and strategies oriented to evaluation. The mentioning of individual differences was present across the reports of both students and tutors. Conclusions: Students use a rich variety of strategies to face the challenges of clinical learning. Both students and tutors recognize that the learning approaches and strategies vary according the nature of the task and individual differences. The responses of students bring particular knowledge of the approaches used for the theoretical and practical integration and delve into the social dimension of learning.

(Rev Med Chile 2015; 143: 1395-1404)

Key words: Clinical competence; Students, medical; Study characteristics.

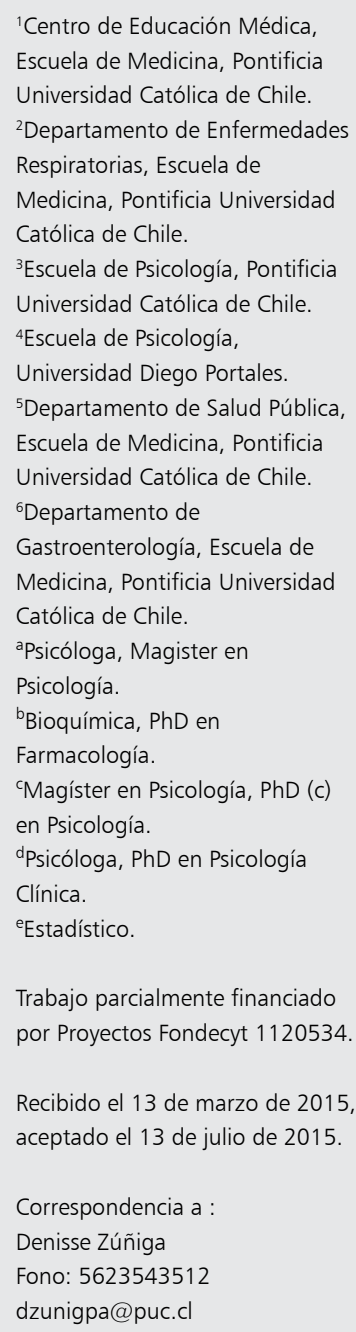

Recibido el 13 de marzo de 2015 aceptado el 13 de julio de 2015.

Correspondencia a :

Denisse Zúñiga

Fono: 5623543512

dzunigpa@puc.cl

L

a metodología de enseñanza-aprendizaje de la medicina de pregrado se caracteriza por cambiar gradualmente desde una formación principalmente teórica hacia una de carácter práctico $^{1-3}$. Las escuelas ofrecen a sus estudiantes oportunidades de aprendizaje experiencial en contacto directo con pacientes, bajo la supervisión de tutores clínicos, y en un ambiente físico y social lo más parecido al que encontrarán cuando sean profesionales ${ }^{1}$. La aproximación a la clínica (en escenarios simulados o reales) se inicia normalmente en 3er año y se consolida en el internado $\left(6^{\circ}\right.$ y $\left.7^{\circ}\right)$. Desde el punto de vista de la enseñanza como del aprendizaje, el período entre $4^{\circ}$ y $5^{\circ}$ año es un tiempo de transición e implica un gran desafío para las escuelas de Medicina y sus estudiantes ${ }^{4,5}$. 
En Chile, así como en muchos países de Norteamérica y Europa, los estudiantes que ingresan a medicina suelen ser académicamente excelentes ${ }^{6}$. La mayoría reporta estilos de aprendizaje abstracto-pasivo (asimilador) ${ }^{6,7}$, que caracterizan a las personas capaces de asimilar grandes cantidades de información y entender conceptos abstractos. Los estilos de aprendizaje de los estudiantes de medicina de la Pontificia Universidad Católica de Chile (PUC) son semejantes a los de la Universidad de Concepción y a los de la Universidad de Brasiliaa ${ }^{7,8}$. Sin embargo, la proporción de aprendices activos (convergentes y acomodadores) es menor en los estudiantes chilenos que en los estudiantes de medicina norteamericanos, cuya especialidad es la aplicación práctica de los contenidos ${ }^{9,10}$.

El predominio de asimiladores supone una transición desafiante. En este período, los estudiantes deberán adaptar sus modos de aprender la medicina considerando la complejidad de las nuevas condiciones que se les propone: aprender a aprender activamente junto a otros y a utilizar los conocimientos previos para interpretar las nuevas experiencias obtenidas en la clínica. Por tanto, no sólo deben demostrar dominio conceptual, también desarrollar la capacidad de integrar, de hacer y de comunicarse empática y profesionalmente con los pacientes ${ }^{1-3,11-13}$.

Aunque abundan los estudios respecto de las estrategias y estilos de aprendizaje de estudiantes en contextos lectivos ${ }^{14-16}$, poco se sabe respecto de cómo se aproximan al aprendizaje en contextos prácticos ${ }^{17}$. Una dificultad es la escasez de instrumentos específicos para identificar las estrategias para el aprendizaje de la clínica. Como una forma de contribuir al conocimiento en este punto, realizamos un proyecto FONDECYT que buscó conocer la percepción de estudiantes y docentes clínicos respecto de cómo aprenden los estudiantes de medicina en la transición hacia la clínica ${ }^{17}$.
Este estudio reporta los resultados derivados del estudio cualitativo que indagó respecto de uno de los temas emergentes de mayor riqueza: estrategias de aprendizaje.

El objetivo del estudio es identificar las estrategias de aprendizaje usadas por los estudiantes de medicina durante la formación clínica inicial reportadas por estudiantes y docentes clínicos.

\section{Materiales y Métodos}

\section{Diseño de la investigación}

El estudio tuvo un diseño cualitativo, de alcance descriptivo y relacional. La metodología cualitativa está orientada al descubrimiento del objeto de investigación y la construcción del conocimiento fundamentado empíricamente, desde el marco de referencia y discurso de las personas ${ }^{18}$. Utilizamos el enfoque de Teoría Fundada para el análisis de los contenidos, que reconoce la naturaleza social del fenómeno en estudio y asigna al investigador un rol participativo en la construcción de la teoría resultante ${ }^{19}$. Este enfoque permite descubrir, desarrollar y verificar provisionalmente categorías emergentes 'enraizadas' en los datos, que expliquen los fenómenos en estudio a partir de un proceso de recolección y análisis sistemático y en relación recíproca ${ }^{18-20}$.

\section{Participantes}

Participaron 54 estudiantes que cursaban entre $3^{\text {ro }}$ y $6^{\text {to }}$ año de medicina y ocho docentes clínicos con experiencia docente en pregrado en la misma carrera. Los participantes fueron seleccionados por muestreo intencionado. Los criterios de inclusión están indicados en la Tabla 1.

\section{Técnicas de recolección de información}

Se realizaron ocho entrevistas grupales focalizadas con los estudiantes conducidas por dos

Tabla 1. Participantes y técnicas de recolección de información

\begin{tabular}{|lll|}
\hline Participantes & Criterios de inclusión & Técnicas de recolección \\
54 estudiantes de Medicina & - Cursan 3ro a 6to año de carrera & Entrevista grupal focalizada (2 por nivel) \\
(H: 29; M: 25) & - Hombres y mujeres & \\
8 docentes clínicos & - Realizan docencia en 3ro a 6to año de carrera & Entrevista individual semiestructurada \\
(H: $7 ;$ M: 1$)$ & - $\geq 4$ años de experiencia docente & \\
& - Hombres y mujeres & \\
\hline
\end{tabular}

H: Hombres; M: Mujeres. 
investigadores. La información de los docentes clínicos se recolectó mediante entrevistas individuales conducidas por un investigador. Las entrevistas se implementaron en sesiones de 60-90 min entre abril y noviembre del año 2012.

Ambas técnicas de recolección se desarrollaron en base a un guión de preguntas semiestructuradas y abiertas, destinadas a indagar las percepciones que los participantes tenían sobre cuáles son las estrategias que los estudiantes de medicina utilizan para integrar la teoría con la práctica clínica y para comunicarse con los pacientes.

La participación fue voluntaria. Los participantes fueron invitados individualmente vía correo electrónico. Quienes aceptaron participar firmaron un consentimiento informado antes del inicio de la actividad. El consentimiento y el estudio fueron aprobados por el comité de Ética de la Escuela de Medicina (PUC) y FONDECYT $\left(\mathrm{N}^{\circ} 1120534\right)$.

\section{Análisis de los datos}

El análisis se realizó a medida que se recolectaban los datos con el fin de mejorar los guiones de la entrevista. La recolección continuó hasta no obtener información nueva. Los registros de audio de las sesiones se transcribieron y analizaron según los procedimientos de la Teoría Fundada en el nivel axial ${ }^{20}$. Primero, los datos fueron agrupados en categorías temáticas (codificación abierta). Esta etapa se apoyó con el uso del programa ATLAS.ti versión 7.1@. Luego, se realizó una codificación axial, que establece relaciones entre las categorías de la codificación abierta generando modelos comprensivos sobre el objeto de estudio ${ }^{20}$. La generación de categorías se orientó por el criterio de descripción densa, es decir, procurando comprender e interpretar de manera contextualizada las respuestas. Con el propósito de asegurar la validez de los resultados, se empleó la estrategia de triangulación, que implica confirmar las categorías emergentes a través del acuerdo intersubjetivo de los investigadores ${ }^{20}$.

\section{Resultados}

\section{Estrategias de aprendizaje}

Ante la indagación respecto de cuáles son las estrategias que usan los estudiantes de medicina para integrar los conocimientos teóricos con la práctica clínica y para comunicarse con los pacientes, surgieron cuatro categorías en el discurso de los participantes: estrategias orientadas al aprendizaje teórico, orientadas al aprendizaje práctico, estrategias de aprendizaje orientadas a la integración teórico-práctica y orientadas por la evaluación (Tabla 2).

Las estrategias orientadas al aprendizaje teórico

Tabla 2. Estrategias utilizadas por los estudiantes de medicina para el aprendizaje clínico, según la percepción de estudiantes y docentes clínicos

\begin{tabular}{|c|c|c|c|}
\hline \multicolumn{2}{|c|}{ Estrategias de aprendizaje } & \multicolumn{2}{|c|}{ Percepción de } \\
\hline Categoría & Subcategoría & Estudiantes & Docentes \\
\hline \multirow[t]{3}{*}{ Orientadas al aprendizaje teórico } & Estudiar & $\bullet$ & $\bullet$ \\
\hline & Buscar información & - & - \\
\hline & Asistir a clases & • & \\
\hline \multirow[t]{7}{*}{ Orientadas al aprendizaje práctico } & Ejercitar en forma individual & • & $\bullet$ \\
\hline & Apoyarse en el par & • & • \\
\hline & Apoyarse en internos y residentes & • & • \\
\hline & Practicar con pacientes & • & • \\
\hline & Observar y preguntar al tutor & • & \\
\hline & Interactuar con el equipo de salud & - & \\
\hline & Aprender por ensayo y error & - & \\
\hline \multirow[t]{3}{*}{ Orientadas a la integración teórico-práctica } & Aprender desde textos y pacientes & $\bullet$ & \\
\hline & Estudiar para argumentar las respuestas & • & \\
\hline & Estudiar para tomar decisiones clínicas & $\bullet$ & \\
\hline \multirow[t]{2}{*}{ Orientadas a la evaluación } & Estudiar por la nota & • & \\
\hline & Anticipar preguntas de prueba o paso práctico & • & \\
\hline
\end{tabular}


se refieren a técnicas de estudio (individual o junto a otros) y a la búsqueda de información para la adquisición del conocimiento. Adicionalmente, los estudiantes mencionan el asistir a clases como otra estrategia de aprendizaje orientada al aprendizaje teórico.

Estrategias orientadas al aprendizaje práctico es la categoría más densamente descrita por estudiantes y docentes, y está caracterizada principalmente por la consideración de "un otro" como recurso de aprendizaje. Por ejemplo, apoyarse en pares de distinto nivel de formación y el practicar con pacientes. Los estudiantes mencionan otras tres subcategorías de estrategias de aprendizaje y que no están presentes en el discurso de los docentes: observar y preguntar al tutor sobre procedimientos y destrezas, interactuar con el equipo de salud de los distintos campos clínicos, tomar conciencia del error y aprender por ensayo-error desde su propia experiencia clínica (Tabla 2).

Las estrategias de aprendizaje orientadas a la integración teórico-práctica emergen sólo en el discurso de los estudiantes. Estas estrategias tributan en una zona de intersección entre el aprendizaje del conocimiento teórico y el conocimiento práctico. Estudiar de manera combinada de textos y directamente de los pacientes, y prepararse para argumentar respuestas y/o tomar decisiones clínicas son acciones que apuntan a un modo de saber "pensar" y "hacer" en la práctica clínica.

Las estrategias orientadas por la evaluación se relacionan con la preparación de los estudiantes considerando las calificaciones o los métodos de evaluación que deberán enfrentar. Por ejemplo, estudiar por la nota o anticipando preguntas de prueba o paso práctico.

Citas representativas de todas las categorías y subcategorías de estrategias de aprendizaje reportadas por los estudiantes y los docentes clínicos se muestran en la Tabla 3.

\section{Diferencias individuales}

Las respuestas de estudiantes y docentes también revelan, que las distintas estrategias de aprendizaje usadas por los estudiantes están mediadas por diferencias individuales. La motivación por determinados conocimientos, el nivel de activación que produce el contenido y/o las actividades y la necesidad del otro como recurso de aprendizaje, fueron tres subcategorías de respuesta que dan cuenta de diferencias entre los estudiantes.
Las categorías de estrategias de aprendizaje hasta aquí reportadas, fueron progresivamente integradas y organizadas en torno a dos temáticas o modelos resultantes: la dinámica de las estrategias de aprendizaje de la clínica y desarrollo de las estrategias de aprendizaje en la transición de la clínica en el contexto de la formación médica.

\section{Dinámica de las estrategias de aprendizaje de la clínica}

La Figura 1 representa un modelo que organiza el discurso de estudiantes y docentes clínicos respecto de cómo operan las estrategias de aprendizaje usadas por los estudiantes de medicina en la transición al aprendizaje clínico. Este modelo se enmarca en el contexto del proceso de enseñanza-aprendizaje (teórico y práctico) característico de la transición hacia la clínica entre $3^{\circ}$ y $6^{\circ}$ de medicina. De acuerdo al modelo, las estrategias se orientan según el tipo de aprendizaje que los estudiantes deban enfrentar. A su vez, e independiente de su orientación, se pueden distinguir estrategias de aprendizaje en las que el estudiante puede interactuar con el objeto de aprendizaje de dos modos: individual y social. Por una parte, existen estrategias en las que el estudiante interactúa de manera directa e individual con el contenido de aprendizaje, por ejemplo, la búsqueda de información, la sistematización y consolidación de contenidos. Por otra, se identifican estrategias de aprendizaje caracterizadas por interacciones con un otro que facilitan o promueven el desarrollo del conocimiento. Por ejemplo, aprender a través de la interacción con internos y residentes para resolver dudas y obtener retroalimentación. El repertorio de estrategias descritas tiene como metas finales el dominio del conocimiento (saber y hacer) para desempeñarse como estudiante y como médico (Figura 1).

\section{Desarrollo de las estrategias de aprendizaje en la transición de la clínica en el contexto de la formación médica}

La Figura 2 intenta complementar la comprensión de los resultados. El repertorio de estrategias de aprendizaje se representa como una espiral que crece gradualmente dada las nuevas condiciones que el contexto de formación propone al estudiante durante la transición a la clínica. Estas estrategias están situadas también sobre dos resultados de aprendizaje claves en la formación: el logro de los 


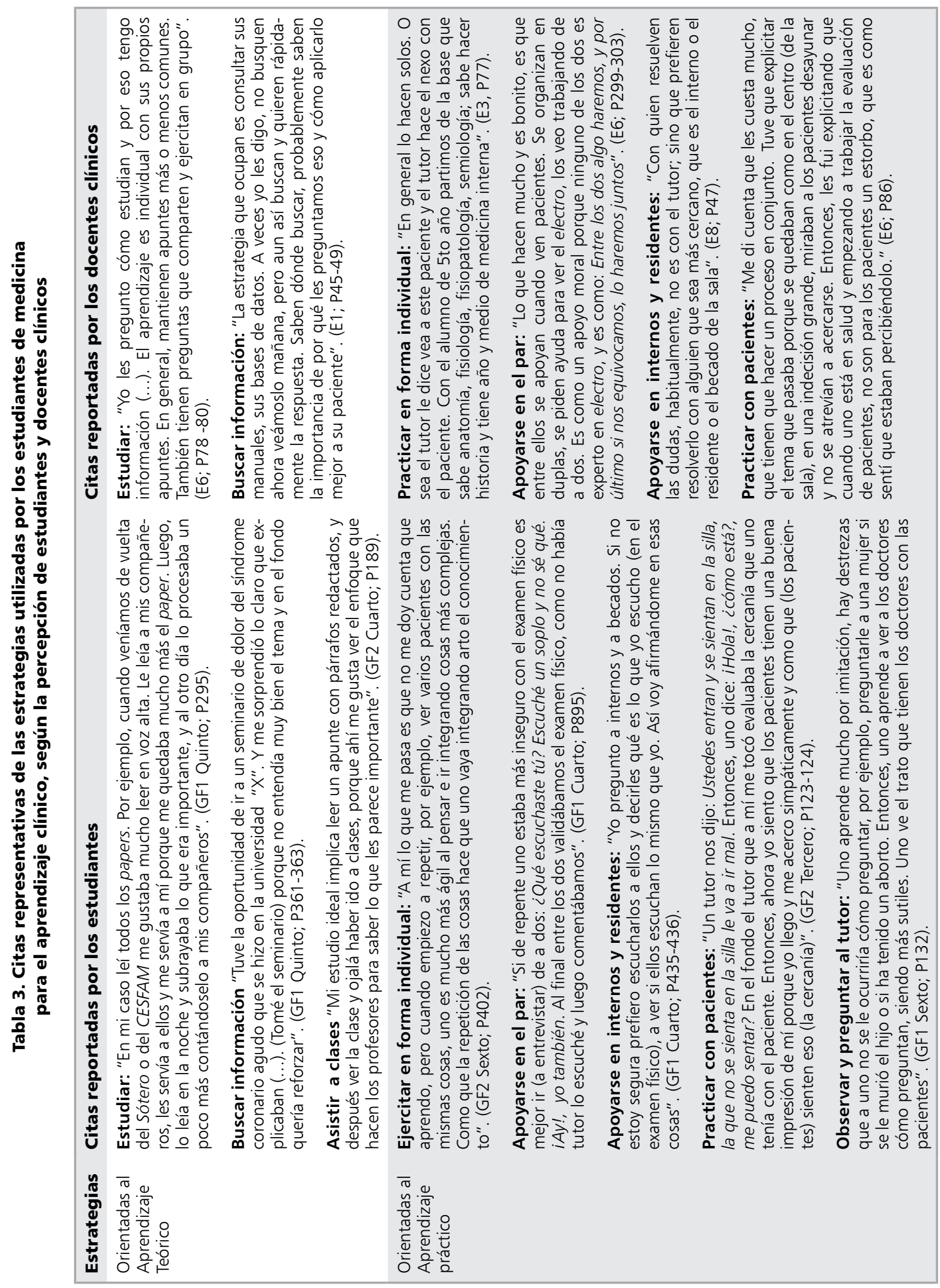




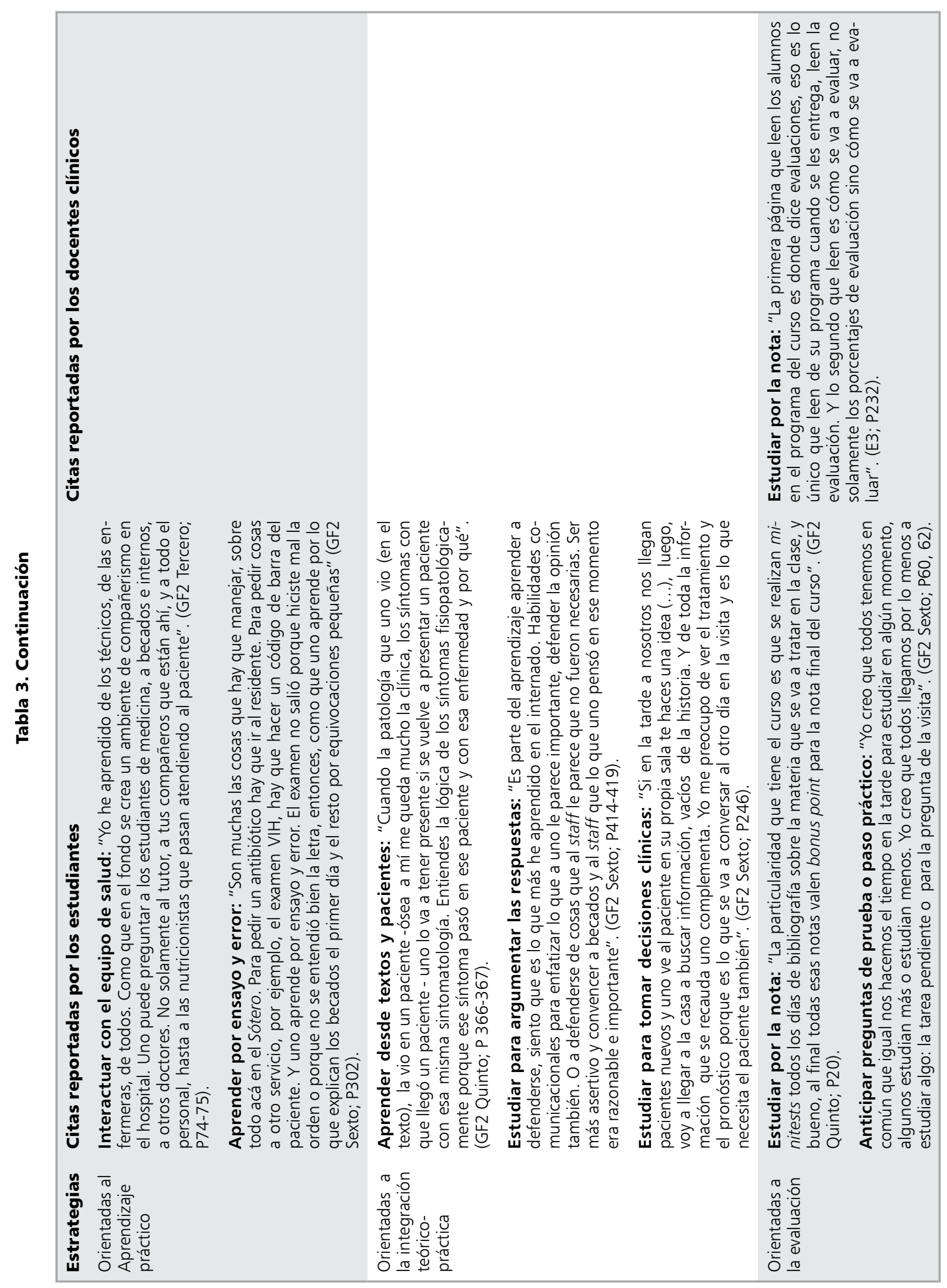



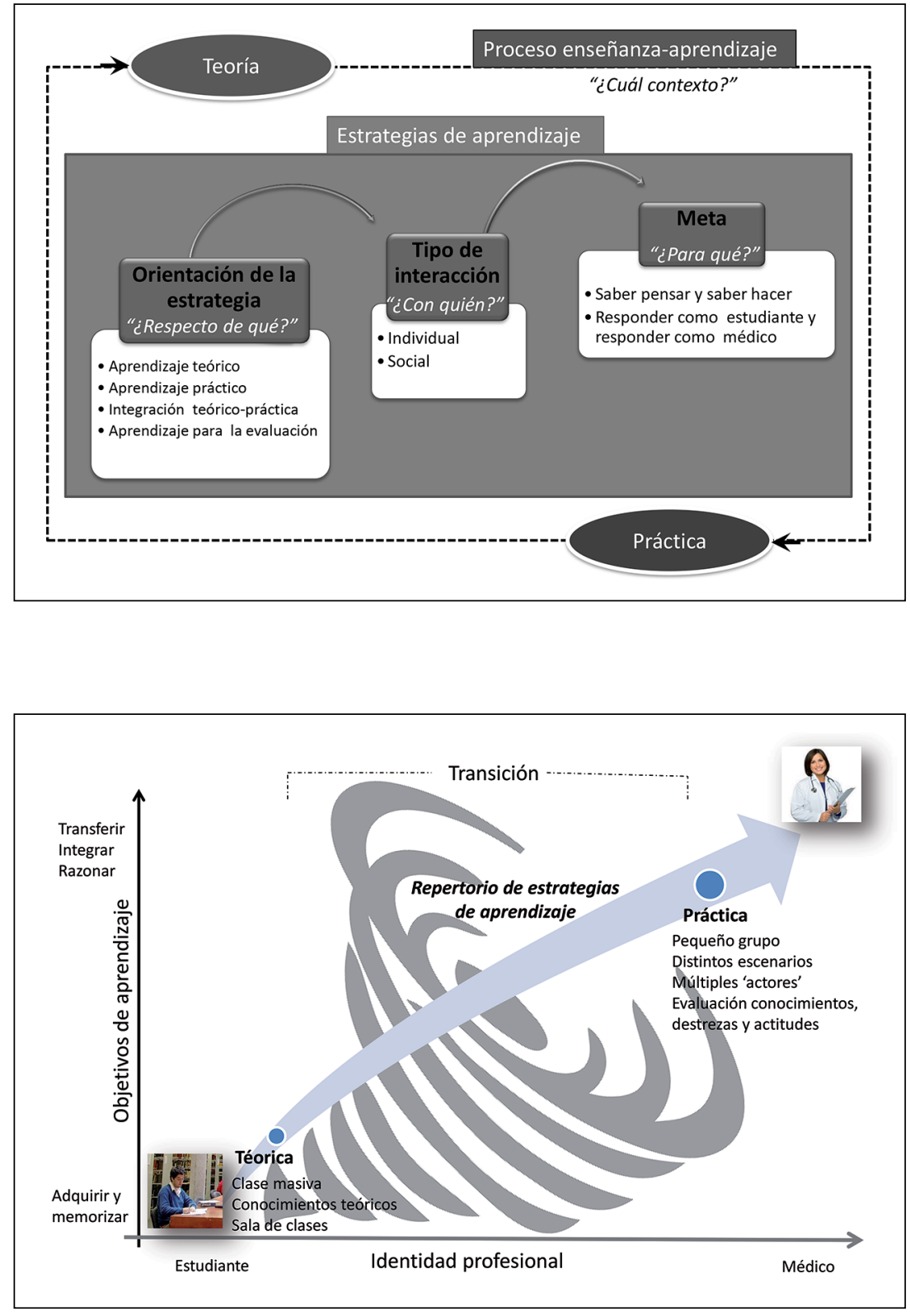

Figura 1. Modelo relacional de las estrategias de aprendizaje de la clínica.
Figura 2. Propuesta de la dinámica de desarrollo de las estrategias de aprendizaje de la clínica. objetivos de aprendizaje definidos en el currículo y el desarrollo de la identidad profesional. Las estrategias de aprendizaje orientadas al aprendizaje práctico; por ejemplo, practicar con pacientes e interactuar con el equipo de salud en escenarios clínicos reales, contribuyen al desarrollo de la identidad y al acercamiento gradual del estudiante al ejercicio profesional futuro (Figura 2).

\section{Discusión}

El objetivo del estudio fue identificar las estrategias de aprendizaje usadas por los estudiantes de medicina durante la formación inicial de la clínica. A partir de las respuestas de estudiantes y docentes clínicos es posible reconocer que ambos 'actores' claves del proceso de enseñanza-aprendizaje 
dan cuenta de una gran variedad de estrategias y que éstas van más allá de las relacionadas con hábitos de estudio y la práctica con pacientes. La perspectiva de los estudiantes, además, nos revela un conocimiento más específico sobre estrategias relacionadas con la integración teórico-práctica y el valor de la dimensión social del aprendizaje. La integración de las estrategias de aprendizaje identificadas, a través del marco de teoría fundada adoptado, permitiría dar cuenta y proponer modelos comprensivos respecto de cómo operan estas estrategias en el contexto de enseñanza del aprendizaje clínico, en específico y cómo se desarrollan y complejizan en el contexto de la formación médica más general.

Es necesario destacar que el dinamismo y complejidad de las estrategias de aprendizaje de los estudiantes que se expresan en estos resultados, podrían considerarse expresiones de una 'meta-estrategia' ${ }^{21}$. El aprendizaje (probablemente implícito) de los objetivos de los diferentes niveles de formación, va contribuyendo al uso cada vez más pertinente y versátil (en el caso ideal) de las estrategias de aprendizaje. Si las estrategias utilizadas en el ciclo básico se caracterizaban por la memorización de contenidos, las estrategias más avanzadas debieran permitir el desarrollo de un razonamiento organizado, la integración y la transferencia de conocimientos a nuevas situaciones clínicas. Este aprendizaje de orden superior implica, como lo señalan algunos estudios, aprender a gestionar las estrategias de acuerdo al nivel de formación, a los objetivos de aprendizaje y considerando incluso las propias expectativas que tenga el estudiante sobre su aprendizaje y el contexto $^{22-26}$.

Que algunas de las estrategias de aprendizaje inicial de la clínica reportadas resultaran 'invisibles' a ojos del docente puede deberse a varias razones: que se despliegan fuera del ámbito de acción docente, o que las estrategias apuntan al desarrollo de procesos como el razonamiento clínico o la integración del conocimiento que no son posibles de observar tan directamente. Una tercera posibilidad, que hace sentido a través de los dos modelos propuestos, tiene que ver con que la complejidad que alcanzan las estrategias de los estudiantes está a la altura de la complejidad percibida en el contexto en el que ocurre su aprendizaje. La complejidad del contexto que resulta desafiante para los estudiantes, al punto de amenazar el bienestar en algunos casos, es obvia para los docentes ${ }^{17,27,28}$.

En este sentido la diversidad que propone el contexto clínico cumple con la tarea de tensionar el modo previo de funcionar que tenía el estudiante desde la secundaria y lo 'empuja' no sólo a perfeccionarlo, sino a desplegar nuevos modos de aprender, involucrándose integralmente en la experiencia para alcanzar los objetivos de aprendizaje y construir su identidad profesional ${ }^{4,5,21,28-33}$.

Desde el punto de vista conceptual y empírico, encontramos en la perspectiva sociocultural una propuesta conforme al modo en que estamos entendiendo el aprendizaje de nuestros estudiantes $^{34-36}$. Si el aprendizaje es desarrollo y el desarrollo es transformación, la transformación del estudiante que apreciamos en la transición hacia la clínica ocurre situadamente y con otros, incluso el aprendizaje individual ocurre socialmente. La relevancia de "lo social" y "contextual" está presente también en la importancia de la participación activa, el aprendizaje colaborativo, y el aprendizaje basado en la práctica ${ }^{37-40}$.

\section{Limitaciones}

Una de las limitaciones de este estudio es que informa los resultados de una escuela de medicina, lo que podría limitar la generalización de sus conclusiones. Sin embargo, las observaciones gruesas que resaltan la importancia de los aspectos sociales y contextuales para el aprendizaje son muy probablemente comunes a la formación de los médicos. Para determinar si hay variantes en las estrategias específicas usadas por los estudiantes, sería interesante contrastar estos resultados y ponerlos en perspectiva con los hallazgos de otras escuelas y proponer análisis específicos según los distintos niveles de formación. Un avance respecto de las estrategias de aprendizaje según nivel, desde la metodología cuantitativa, es reportado en un artículo que los autores presentaron a Revista Médica para su publicación.

\section{Conclusión}

Los estudiantes de medicina emplean una amplia variedad de estrategias para enfrentar los desafíos de la formación clínica inicial. Estudiantes 
y docentes reconocen que éstas varían de acuerdo a la naturaleza de la tarea y a diferencias individuales. Las respuestas de estudiantes nos revelan un conocimiento específico sobre las estrategias usadas para la integración teórico-práctica y más profundamente respecto de la dimensión social del aprendizaje en contexto clínico.

Agradecimientos: Queremos agradecer a todos y a cada uno de los estudiantes de 3ero a 6to de medicina del año 2012 y a los docentes clínicos, por aceptar participar en el estudio y compartir sus experiencias con nosotros. Humildemente esperamos que los resultados del estudio, a la luz de la teoría, otorguen nuevo sentido a sus prácticas.

\section{Referencias}

1. Cooke M, Irby D, O’brien B. Educating Physicians. A call for reform of medical school and residency, NY. Jossey-Bass; 2010.

2. Sánchez I, Riquelme A, Moreno R, Mena B, Dagnino J, Grebe G. Revitalizing medical education: the school of medicine at the Pontificia Universidad Católica de Chile. Clin Teacher 2008; 5: 57-61.

3. Martinic S, Moreno R, Muller M, Pimentel F, Ritthershaussen, Calderón M, Cabezas H. Análisis comparativo del componente de práctica en el currículo de formación profesional de médicos y profesores en la Pontificia Universidad Católica de Chile. Estudios Pedagógicos 2014; XI (1): 179-96.

4. Teunissen P, Westerman M. Opportunity or Threat: the ambiguity of the consequences of transitions in medical education. Med Educ 2011; 45: 51-9.

5. Prince KJ, Boshuizen HP, Ven Der Vleuten CPM, Scherpbier A. Students' opinions about their preparation for clinical practice. Med Educ 2005; 39 (7): 704-12.

6. Bitran $M$, Zúñiga $D$, Lafuente $M$, Viviani $P$, Mena B. Tipos Psicológicos y Estilos de Aprendizaje de los Estudiantes que Ingresan a Medicina de la Pontificia Universidad Católica de Chile. Rev Med Chile 2003; 131 (9): 1067-78.

7. Palacios S, Matus O, Soto A, Ibáñez P, Fasce E. Estilos de aprendizaje en primer año de medicina según cuestionario Honey-Alonso. Publicación preliminar. Rev Educ Cienc Salud 2006; 3: 89-94.

8. Sobral D. Diagnostic ability of medical students in relation to their learning characteristics and pre-clinical background. Medical Education 1995; 29: 278-82.

9. Plovnick M. Primary Care Career Choices and Medical
Student Learning Styles. Journal Medical Education 1975; 50: 849-55.

10. Urry L. Integrating concepts of cognitive or learning style: A review with attention to psychometric standards. Otawa, ON: Canadian College of Health Service Executives. 1987.

11. CNA. Perfil profesional y criterios para la acreditación de carreras de medicina. http://www.acreditaccion.cl/ criterios/CriteriosMedicinaFINAL.pdf (consultado el 26 de junio de 2014).

12. Comité de Educación Superior, de la Academia Chilena de Medicina. Situación Actual de la Educación Médica en Chile. Rev Med Chile 2009; 137: 709-12.

13. Rosselot E. Planificando la educación médica para las próximas décadas. Rev Med Chile 2001; 129 (12): 1473 8 .

14. Kolb DA, Boyatzis R, Mainemelis C. Experiential learning theory: Previous research and new directions. In: Sternberg R, Zhang L (Eds.), Perspectives on cognitive learning, and thinking styles: 228-47. Mahwah, NJ: Erlbaum; 2001.

15. Coffield F, Moseley D, Hall E, Ecclestone K. Learning styles and pedagogy in post-16 learning. A systematic and critical review. Learning and Skills.London; Cromwell Press Ltd; 2004.

16. Bitran $M$, Zúñiga $D$, Lafuente $M$, Viviani $P$, Mena B. Tipos Psicológicos y Estilos de Aprendizaje de los Estudiantes que Ingresan a Medicina de la Pontificia Universidad Católica de Chile. Rev Med Chile 2003; 131 (9): 1067-78.

17. Bitran M, Zúñiga D, Leiva I, Calderón M, Tomicic A, Padilla $\mathrm{O}$, et al. ¿Cómo aprenden los estudiantes de medicina en la transición hacia el ciclo clínico? Estudio cualitativo de las percepciones de estudiantes y docentes acerca del aprendizaje inicial de la clínica. Rev Med Chile 2014; 142: 723-31.

18. Krause M, Cornejo M, Radovic J. Diseño de estudios cualitativos. Apunte MINSAL. Santiago; MINSAL; 1998.

19. Flick U. Introducción a la investigación cualitativa. Madrid; Morata; 2007.

20. Straus A, Corbin J. Basics of Qualitative Research: Grounded Theory Procedures and Techniques. Thousand Oaks, Calif; Sage; 1990.

21. Meyer JHF, Dunne TT. Study approaches of nursing students: effects of an extended clinical context. Med Educ 1991; 25 (6): 401-7.

22. Meyer J. Study orchestration: the manifestation, interpretation and consequences of contextualised approaches to studying. Higher Education 1991; 22 (3): 297-316.

23. Chipchase L, Buttrum P, Dunwoodie R, Hill A, Mandru- 
siak A, Moran M. Characteristics of student preparedness for clinical learning: clinical educator perspectives using the Delphi approach. Med Educ 2012; 12: 1-9.

24. Berbén A, Pichardo M, De la Fuente J. Relaciones entre preferencias de la enseñanza y enfoques de aprendizaje de los universitarios. Infancia y Aprendizaje 2007; 30 (4): 537-50.

25. Mayor J, Suengas A, González MJ. Estrategias metacognitivas, aprender a aprender y aprender a pensar. Madrid; Sintesis SA; 1995.

26. Pozo JI. Metacognición y estrategias de aprendizaje. Madrid; Santillana; 1999.

27. Dahline ME, Runeson B. Burnout and psychiatric morbidity among medical students entering clinical training: a three year prospective questionnaire and interview-based study. BMC Med Educ 2007; 7: 6.

28. Benítez C, Quintero J, Torres R. Prevalencia de riesgo de trastornos psiquiátricos en estudiantes de pregrado de la Escuela de Medicina de la Pontificia Universidad Católica de Chile. Rev Med Chile 2001; 129 (2): 173-8.

29. Helmich E, Bolhuis S, Dornan T, Laan R, Koopmans R. Entering medical practice for the very first time: emotional talk, meaning and identity development. Med Educ 2012; 46: 1074-87.

30. Dobkin P, Balass S. Multiple influences contribute to medical students' well-being and identity formation. Med Educ 2014; 48: 340-8.

31. Kolb A, Kolb D. The learning way. Meta-cognitive aspects of experiential learning. Simulation \& Gaming 2009; 40 (3): 297-325.

32. Kallio E. Integrative thinking is the key: An evaluation of current research into the development of adult thinking.
Theory and Psychology 2011; 21 (6): 785-801.

33. Greenberg L, Blatt B. Sucessfully Negotiating the clerkship years of medical schools: A guide for medical students. Implications for residents and faculty. Acad Med 2010; 85 (4).

34. Yardley S, Teunissen P and Dornan T. AMEE guides: Theories in Medical Education. Experiential Learning AMEE guide No. 63. Med Teach 2012; 34: e102-15.

35. Durning S, Artino A. Situativity theory: A perspective on how participants and the environment can act. Med Teach 2011; 33: 188-99.

36. Van Der Zwet J, Zwietering P, Teunissen P, Van Der Vleuten C, Scherbier A. Workplace learning from a socio-cultural perspective: creating developmental space during the general practice clerkship. Adv Health Sci Educ Theory Pract 2011; 16 (3): 359-73.

37. Wooley N, Jarvis Y. Situated cognition and cognitive apprenticeship: A model for teaching and learning clinical skills in a technologically rich and authentic learning environment. Nurse Education Today 2007; 27: 73-9.

38. Lave J, Wenger E. Situated learning: Legitimate peripheral participation. Cambridge; Cambridge University Press; 1991.

39. Nelson C, Frosch Z, Lapin J, Kogan J. Facilitating the clerkship transition through near peer led student reports. Med Educ 2014; 48: 522-48.

40. Dornan T, Tan N, Boshuizen H, Gick R, Isba R, Mann $\mathrm{K}$, Scherpbier A, Spencer J, Timmins E. How and what do medical sudents learn in clerkships? Experienced based learning. Adv Health Sciences Educ 2015; 19 (5): 721-49. 